H. Morikawa

Nagoya Math. J.

Vol. 63, (1976), 163-171

\title{
A NOTE ON HOLOMORPHIC MATRIC AUTOMORPHIC FACTORS WITH RESPECT TO A LATTICE IN A COMPLEX VECTOR SPACE
}

\author{
Dedicated to the memory of Taira Honda
}

\section{HISASI MORIKAWA}

1. A holomorphic $n \times n$-matric automorphic factor with respect to a lattice $L$ in $C^{\theta}$ means a system of holomorphic $n \times n$-matrices $\left\{\mu_{\alpha}(z) \mid \alpha \in L\right\}$ such that

$$
\begin{aligned}
& \operatorname{det} \mu_{\alpha}(z) \neq 0 \quad \text { everywhere on } C^{g}, \\
& \mu_{\alpha+\beta}(z)=\mu_{\alpha}(z+\beta) \mu_{\beta}(z) \quad(\alpha, \beta \in L) .
\end{aligned}
$$

This is nothing else than the condition of a group action of $L$ on $\boldsymbol{C}^{g} \times \boldsymbol{C}^{n}$;

$$
(z, u) \longrightarrow\left(z+\alpha, \mu_{\alpha}(z) u\right) \quad(\alpha \in L) \text {. }
$$

The quotient $\boldsymbol{E}_{\mu}=\boldsymbol{C}^{g} \times \boldsymbol{C}^{n} / L$ by this group action of $L$ is a holomorphic vector bundle of rank $n$ over the complex torus $C^{g} / L$. Holomorphic vector bundles over the complex torus $C^{g} / L$ are always constructed by this way, since holomorphic vector bundles over $C^{g}$ are trivial.

Denoting

$$
\omega_{\alpha}(z)=\mu_{\alpha}(z)^{-1} d \mu_{\alpha}(z) \quad(\alpha \in L)
$$

we get a system of $n \times n$-matric connections satisfying

$$
\begin{gathered}
d \omega_{\alpha}(z)+\omega_{\alpha}(z) \wedge \omega_{\alpha}(z)=0 \\
\omega_{\alpha+\beta}(z)=\omega_{\alpha}(z)+\mu_{\alpha}(z)^{-1} \omega_{\beta}(z+\alpha) \mu_{\alpha}(z) \quad(\alpha, \beta \in L) .
\end{gathered}
$$

In the present short note we shall characterize matric automorphic factors $\left\{\mu_{\alpha}(z) \mid \alpha \in L\right\}$ such that

i) the associated vector bundle $E_{\mu}$ is simple and ii)

Received February 27, 1976. 


$$
\mu_{\alpha}(z+\beta) \mu_{\alpha}(z)^{-1} \quad(\alpha, \beta \in L)
$$

are constant matrices.

Proposition 1. Let $\left\{\mu_{\alpha}(z) \mid \alpha \in L\right\}$ be a holomorphic $n \times n$-matric automorphic factor with respect to a lattice $L$ in $C^{g}$, and let $\omega_{\alpha}(z)$ be the integrable connections given by

$$
\omega_{\alpha}(z)=\mu_{\alpha}(z)^{-1} d \mu_{\alpha}(z) \quad(\alpha \in L) .
$$

Then following three conditions are equivalent each other,

(5) $\omega_{\alpha}(z)=\sum A_{\alpha \ell} d z_{\ell}$ with constant matrices $A_{\alpha \ell} \quad(\alpha \in L ; 1 \leq \ell \leq g)$.

(6) $\mu_{\alpha}(z)=\mu_{\alpha}(0) \exp \left\{\sum A_{\alpha \ell} z_{\ell}\right\}$ with constant matrices $A_{\alpha \ell}$ satisfying $\left[A_{\alpha \ell}, A_{\alpha h}\right]=0 \quad(\alpha \in L ; 1 \leq \ell, h \leq g)$,

(7) $\mu_{\alpha}(z+\beta) \mu_{\alpha}(z)^{-1} \quad(\alpha, \beta \in L)$ are constant matrices .

Proof. If we assume (5), we have $\omega_{\alpha}(z) \wedge \omega_{\alpha}(z)=0$ and thus $\left[A_{\alpha \ell}, A_{\alpha h}\right]$ $=0 \quad(\alpha, \beta \in L ; 1 \leq \ell, h \leq g)$. By virtue of this commutativity, putting $\tilde{\mu}_{\alpha}(z)=\mu_{\alpha}(0) \exp \left\{\sum A_{\alpha \ell} z_{\ell}\right\}$, we have

$$
\tilde{\mu}_{\alpha}(z)^{-1} d \tilde{\mu}_{\alpha}(z)=\omega_{\alpha}(z)=\mu_{\alpha}(z)^{-1} d \mu_{\alpha}(z) \quad(\alpha \in L) .
$$

Since $\tilde{\mu}_{\alpha}(0)=\mu_{\alpha}(0)$, we have $\tilde{\mu}_{\alpha}(z)=\mu_{\alpha}(z)(\alpha \in L)$. If we assume (6), then

$$
\mu_{\alpha}(z+\beta) \mu_{\alpha}(z)^{-1}=\mu_{\alpha}(0) \exp \left\{\sum A_{\alpha \ell} \beta_{\ell}\right\} \mu_{\alpha}(0)^{-1} \quad(\alpha, \beta \in L)
$$

are constant matrices. Let us show (7) from (5). From the equation $d\left(\mu_{\alpha}(z+\beta) \mu_{\alpha}(z)^{-1}\right)=0$ it follows

$$
\begin{aligned}
\omega_{\alpha}(z+\beta)-\omega_{\alpha}(z) & =\mu_{\alpha}(z+\beta)^{-1} d \mu_{\alpha}(z+\beta)-\mu_{\alpha}(z)^{-1} d \mu_{\alpha}(z) \\
& =\mu_{\alpha}(z+\beta)^{-1} d\left(\mu_{\alpha}(z+\beta) \mu_{\alpha}(z)^{-1}\right) \mu_{\alpha}(z)=0,
\end{aligned}
$$

and thus $\omega_{\alpha}(z+\beta)=\omega_{\alpha}(z)(\alpha, \beta \in L)$. By virtue of compactness of $C^{g} / L$ $\omega_{\alpha}(z)$ can be written

$$
\omega_{\alpha}(z)=\sum A_{\alpha \ell} d z_{\ell}
$$

with constant matrices.

Proposition 2. Let $\left\{\mu_{\alpha}(0) \mid \alpha \in L\right\},\left\{A_{\alpha, \ell} \mid \alpha \in L, 1 \leq \ell \leq g\right\}$ be two systems of constant $n \times n$-matrices such that $\operatorname{det} \mu_{\alpha}(0) \neq 0,\left[A_{\alpha \ell}, A_{\alpha h}\right]=0$ $(\alpha \in L ; 1 \leq \ell, h \leq g)$. Then $\left\{\mu_{\alpha}(0) \exp \left\{\sum A_{\alpha \ell} z_{\ell}\right\} \mid \alpha \in L\right\}$ is a holomorphic $n \times n$-matric automorphic factor with respect to $L$, if and only if 


$$
A_{\alpha+\beta, \ell}=\mu_{\beta}(0)^{-1} A_{\alpha \ell} \mu_{\beta}(0)+A_{\beta \ell} \quad(\alpha, \beta \in L ; 1 \leq \ell \leq g),
$$

$$
\left[\mu_{\beta}(0)^{-1} A_{\alpha \ell} \mu_{\beta}(0), A_{\beta h}\right]=0 \quad(\alpha, \beta \in L ; 1 \leq \ell, h \leq g) .
$$

Proof. Assume that $\left\{\mu_{\alpha}(z)=\mu_{\alpha}(0) \exp \left(\sum A_{\alpha \ell} z_{\ell}\right)\right\}$ is an automorphic factor with respect to $L$. From the relations

$$
\begin{gathered}
\mu_{-\alpha}(z+\alpha) \mu_{\alpha}(z)=I, \quad \omega_{\alpha}(z)=\sum A_{\alpha \ell} d z_{\ell}, \\
\omega_{\alpha+\beta}(z)=\mu_{\alpha}(z)^{-1} \omega_{\beta}(z+\alpha) \mu_{\alpha}(z)+\omega_{\alpha}(z)
\end{gathered}
$$

it follows

$$
\begin{aligned}
\omega_{-\alpha+\beta}(0) & =\omega_{-\alpha+\beta}(z+\alpha)=\mu_{-\alpha}(z+\alpha)^{-1} \omega_{\beta}(z) \mu_{-\alpha}(z+\alpha)+\omega_{-\alpha}(z+\alpha) \\
& =\mu_{\alpha}(z) \omega_{\beta}(z) \mu_{\alpha}(z)^{-1}+\omega_{-\alpha}(z+\alpha) \\
& =\mu_{\alpha}(z) \omega_{\beta}(0) \mu_{\alpha}(z)^{-1}+\omega_{-\alpha}(0)=\mu_{\alpha}(0) \omega_{\beta}(0) \mu_{\alpha}(0)^{-1}+\omega_{-\alpha}(0),
\end{aligned}
$$

and thus

$$
\mu_{\alpha}(0)^{-1} \mu_{\alpha}(z) \omega_{\beta}(0)=\omega_{\beta}(0) \mu_{\alpha}(0)^{-1} \mu_{\alpha}(z) \quad(\alpha, \beta \in L) .
$$

Comparing the coefficients of $z_{\ell} d z_{h}$ in the both sides of

$$
\exp \left\{\sum A_{\alpha \ell} z_{\ell}\right\} \sum A_{\beta h} d z_{h}=\sum A_{\beta h} d z_{h} \exp \left\{\sum A_{\alpha \ell} z_{\ell}\right\}
$$

we have $\left[A_{\alpha \ell}, A_{\beta h}\right]=0(\alpha, \beta \in L ; 1 \leq \ell, h \leq g)$. From the relation $\mu_{\alpha+\beta}(z)$ $=\mu_{\alpha}(z+\beta) \mu_{\beta}(z)$ we have

$$
\begin{aligned}
\mu_{\alpha+\beta}(z) & =\mu_{\alpha+\beta}(0) \exp \left\{\sum A_{\alpha+\beta \ell} z_{\ell}\right\} \\
& =\mu_{\alpha}(0) \exp \left\{\sum A_{\alpha \ell} \beta_{\ell}\right\} \mu_{\beta}(0) \exp \left\{\sum A_{\alpha+\beta \ell} z_{\ell}\right\} \\
& =\mu_{\alpha}(z+\beta) \mu_{\beta}(z) \\
& =\mu_{\alpha}(0) \exp \left\{\sum A_{\alpha \ell}\left(z_{\ell}+\beta_{\ell}\right)\right\} \mu_{\beta}(0) \exp \left\{\sum A_{\beta \ell} z_{\ell}\right\} \\
& =\mu_{\alpha}(0) \exp \left\{\sum A_{\alpha \ell} \beta_{\ell}\right\} \mu_{\beta}(0) \\
& =\exp \left\{\sum \mu_{\beta}(0)^{-1} A_{\alpha \ell} \mu_{\beta}(0) z_{\ell}\right\} \exp \left\{\sum A_{\beta \ell} z_{\ell}\right\}
\end{aligned}
$$

Hence, comparing the coefficients of $z_{\ell}$ and $z_{\ell} z_{h}$ in the both sides of

$$
\exp \left\{\sum A_{\alpha+\beta \ell} z_{\ell}\right\}=\exp \left\{\sum \mu_{\beta}(0)^{-1} A_{\alpha \ell} \mu_{\beta}(0) z_{\ell}\right\} \exp \left\{\sum A_{\beta \ell} z_{\ell}\right\},
$$

respectively, we have

$$
\begin{aligned}
A_{\alpha+\beta \ell}= & \mu_{\beta}(0)^{-1} A_{\alpha \ell} \mu_{\beta}(0)+A_{\beta \ell}, \\
A_{\alpha+\beta \ell} A_{\alpha+\beta h}= & \mu_{\beta}(0)^{-1} A_{\alpha \ell} \mu_{\beta}(0) \mu_{\beta}(0)^{-1} A_{\alpha h} \mu_{\beta}(0) \\
& +\mu_{\beta}(0)^{-1} A_{\alpha \ell} \mu_{\beta}(0) A_{\beta h}+\mu_{\beta}(0)^{-1} A_{\alpha h} \mu_{\beta}(0) A_{\beta \ell}+A_{\beta \ell} A_{\beta n}
\end{aligned}
$$


and thus

$$
A_{\beta \ell} \mu_{\beta}(0)^{-1} A_{\alpha h} \mu_{\beta}(0)=\mu_{\beta}(0)^{-1} A_{\alpha h} \mu_{\beta}(0) A_{\beta \ell} .
$$

Namely $\left[A_{\beta \ell}, \mu_{\beta}(0)^{-1} A_{\alpha h} \mu_{\beta}(0)\right]=0(\alpha, \beta \in L ; 1 \leq \ell, h \leq g)$. Conversely if we assume (8), (9), (10), then putting

$$
\mu_{\alpha}(z)=\mu_{\alpha}(0) \exp \left\{\sum A_{\alpha \ell} z_{\ell}\right\} \quad(\alpha \in L),
$$

we have

$$
\begin{aligned}
\mu_{\alpha}(z & +\beta) \mu_{\beta}(z) \\
& =\mu_{\alpha}(0) \exp \left\{\sum A_{\alpha \ell}\left(z_{\ell}+\beta_{\ell}\right) \mu_{\beta}(0)\right\} \exp \left\{\sum A_{\beta \ell} z_{\ell}\right\} \\
& =\mu_{\alpha}(0) \exp \left\{\sum A_{\alpha \ell} \beta_{\ell}\right\} \mu_{\beta}(0) \exp \left\{\sum \mu_{\beta}(0)^{-1} A_{\alpha \ell} \mu_{\beta}(0) z_{\ell}\right\} \exp \left\{\sum A_{\beta \ell} z_{\ell}\right\} \\
& =\mu_{\alpha}(0) \mu_{\beta}(0) \exp \left\{\sum\left(\mu_{\beta}(0)^{-1} A_{\alpha \ell} \mu_{\beta}(0)+A_{\beta \ell}\right) z_{\ell}\right\} \\
& =\mu_{\alpha+\beta}(0) \exp \left\{A_{\alpha+\beta \ell} z_{\ell}\right\}=\mu_{\alpha+\beta}(z) .
\end{aligned}
$$

\section{COROLLARY 1.}

$$
\begin{aligned}
& \mu_{\alpha}(z)^{-1} \mu_{\beta}(z)^{-1} \mu_{\alpha}(z) \mu_{\beta}(z) \\
& \quad=\exp \left\{\sum\left(A_{\alpha+\beta \ell}-A_{\beta \ell}\right) \alpha_{\ell}-\sum\left(A_{\alpha+\beta \ell}-A_{\alpha \ell}\right) \beta_{\ell}\right\} \quad(\alpha, \beta \in L) .
\end{aligned}
$$

Proof. From the relation $\mu_{\alpha+\beta}(z)=\mu_{\alpha}(z+\beta) \mu_{\beta}(z)=\mu_{\beta}(z+\alpha) \mu_{\alpha}(z)$ it follows

$$
\begin{aligned}
\mu_{\alpha}(z) \mu_{\beta}(z) & =\mu_{\alpha}(0) u_{\beta}(0) \exp \left\{\sum\left(\mu_{\beta}(0)^{-1} A_{\alpha \ell} \mu_{\beta}(0)+A_{\beta \ell}\right) z_{\ell}\right\} \\
& =\mu_{\alpha}(0) \mu_{\beta}(0) \exp \left\{\sum A_{\alpha+\beta \ell} z_{\ell}\right\}, \\
\mu_{\beta}(z) \mu_{\alpha}(z) & =\mu_{\beta}(0) \mu_{\alpha}(0) \exp \left\{\sum\left(\mu_{\alpha}(0)^{-1} A_{\beta \ell} \mu_{\alpha}(0)+A_{\alpha \ell}\right) z_{\ell}\right\} \\
& =\mu_{\beta}(0) \mu_{\alpha}(0) \exp \left\{\sum A_{\alpha+\beta \ell} z_{\ell}\right\}, \\
\mu_{\alpha}(0) \mu_{\beta}(0) & \exp \left\{\sum \mu_{\beta}(0)^{-1} A_{\alpha \ell} \mu_{\beta}(0) \beta_{\ell}\right\} \\
& =\mu_{\alpha+\beta}(0)=\mu_{\beta}(0) \mu_{\alpha}(0) \exp \left\{\sum \mu_{\alpha}(0)^{-1} A_{\beta \ell} \mu_{\alpha}(0) \alpha_{\ell}\right\} .
\end{aligned}
$$

Hence

$$
\begin{aligned}
\mu_{\alpha}(z)^{-1} & \mu_{\beta}(z)^{-1} \mu_{\alpha}(z) \mu_{\beta}(z) \\
& =\mu_{\alpha}(0)^{-1} \mu_{\beta}(0)^{-1} \mu_{\alpha}(0) \mu_{\beta}(0) \\
& =\exp \left\{\sum \mu_{\alpha}(0)^{-1} A_{\beta \ell} \mu_{\alpha}(0) \alpha_{\ell}-\sum \mu_{\beta}(0)^{-1} A_{\alpha \ell} \mu_{\beta}(0) \beta_{\ell}\right\} \\
& =\exp \left\{\sum\left(A_{\alpha+\beta \ell}-A_{\beta \ell}\right) \alpha_{\ell}-\sum\left(A_{\alpha+\beta \ell}-A_{\alpha \ell}\right) \beta_{\ell}\right\}
\end{aligned}
$$

COROLLARY 2. The matric group generated by $\left\{\mu_{\alpha}(z) \mid \alpha \in L\right\}$ is a metabelian group whose derived group is a group of constant matrices.

This is an immediate consequence of Corollary 1. 
2. A holomorphic vector bundle is called to be simple, if its endomorphisms are scaler multiplications. The vector bundle associated with $\left\{\mu_{\alpha}(z) \nmid \alpha \in L\right\}$ is simple, if and only if scaler matrices are only holomorphic matrices $B(z)$ such that

$$
B(z+\alpha) \mu_{\alpha}(z)=\mu_{\alpha}(z) B(z) \quad(\alpha \in L) .
$$

Characterizing a simple vector bundle $E_{\mu}$ such that

$$
\mu_{\alpha}(z)^{-1} d \mu_{\alpha}(z)=\sum_{\ell=1}^{g} A_{\alpha \ell} d z_{\ell} \quad(\alpha \in L)
$$

with constant matrices $A_{\alpha \ell}$, we need the following Clifford theorem,

CLIFFORD THEOREM. Let $G$ be a group and $H$ be a normal subgroup of $G$. Let $V$ be a vector space of finite dimension over a field which is a simple G-module. Then there exists a vector subspace $W$ which is simple $H$-module and elements $g_{11}, \cdots, g_{1 m}, \cdots, g_{r 1}, \cdots, g_{r m}$ in $G$ such that

$$
V=\left(g_{11} W \oplus \cdots \oplus g_{1 m} W\right) \oplus \cdots \oplus\left(g_{r 1} W \oplus \cdots \oplus g_{r m} W\right)
$$

and $g_{i \ell} W$ and $g_{j k} W$ are equivalent $H$-modules if and only if $i=j$.

Proof. Let $W$ be a vector subspace which is a simple $H$-module. Since $H$ is normal in $G$, the images $g W(g \in G)$ are simple $H$-modules, and $V$ is a sum of $g W(g \in G)$. Hence there exist elements $g_{1}^{\prime}, \cdots, g_{p}^{\prime}$ of $G$ such that

$$
V=g_{1}^{\prime} W \oplus \cdots \oplus g_{p}^{\prime} W .
$$

Let $\left\{g_{11}, \cdots, g_{1 m}\right\}$ be the largest subset in $\left\{g_{1}^{\prime}, \cdots, g_{p}^{\prime}\right\}$ such that $g_{1}, W$ $(1 \leq \ell \leq m)$ are equivalent to $W$. Then $V$ is a direct sum of the images of $g_{11} W \oplus \cdots \oplus g_{1 m} W$ by elements of $G$. This completes the proof of Clifford theorem.

LEMMA 1. Let $G$ be a transitive abelian permutation group acting on $\{1,2, \cdots, r\}$. Then $|G|=r$.

Proof. Let $N$ be the stabilizer of letter 1 . Then $|G / N|=r$ and $G$ acts on $G / N$. Two permutation groups $(G,\{1,2, \cdots, r\})$ and $(G, G / N)$ are isomorphic as permutation groups. Since $G$ is abelian, $N$ must be a normal subgroup, and thus any element of $N$ leaves invariant every letter in $\{1,2, \cdots, r\}$. This shows $|G|=|G / N|=r$. 
THEOREM 1. Let $\left\{\mu_{\alpha}(z) \mid \alpha \in L\right\}$ be a holomorphic $n \times n$-matric automorphic factor with respect to a lattice $L$ in $C^{g}$ such that i)

$$
\mu_{\alpha}(z)^{-1} d \mu_{\alpha}(z)=\sum_{\ell=0}^{g} A_{\alpha \ell} d z_{\ell} \quad(\alpha \in L)
$$

with constant matrices $A_{\alpha \ell}$, ii) the associated vector bundle $E_{\mu}$ is simple. Then there exist a sublattice $M$ of $L$ and a line bundle $\mathscr{L}$ over $C^{g} / M$ such that $[L: M]=n$ and $E_{\mu}$ is the direct image of $\mathscr{L}$ with respect to the natural isogeny $C^{g} / M \rightarrow C^{g} / L$.

Proof. We use the following notations:

1: the commutative matric algebra generated by $A_{\alpha, \ell}(\alpha \in L ; 1 \leq \ell \leq g)$ and identity matrix over $\boldsymbol{C}$,

$G$ : the group generated by $\left\{\mu_{\alpha}(0), \exp \left\{\sum A_{\alpha \ell} \beta_{\ell}\right\},(\alpha, \beta \in L)\right\}$ and $G L(n, C) \cap \Lambda$,

$G_{0}$ : the group generated by $\left\{\exp \left\{\sum A_{\alpha \ell} \beta_{\ell}\right\}(\alpha, \beta \in L)\right.$ and $G L(n, C) \cap \Lambda$,

$$
\begin{aligned}
& G_{1}=\left\{g \in G \mid g^{-1} A_{\alpha \ell} g=A_{\alpha \ell}(\alpha \in L ; 1 \leq \ell \leq g)\right\} \\
& L_{1}=\left\{\beta \in L \mid \mu_{\beta}(0)^{-1} A_{\alpha \ell} \mu_{\beta}(0)=A_{\alpha \ell}(\alpha \in L ; 1 \leq \ell \leq g)\right\} .
\end{aligned}
$$

By virtue of (8), (9) and (11) $G_{0}$ is an abelian normal subgroup of $G$ such that $G / G_{0}$ is abelian, and $G_{1}$ is a normal subgroup containing $G_{0}$. Hence $G / G_{1}$ is abelian. If a constant matrix $B$ satisfies

$$
B \mu_{\alpha}(0)=\mu_{\alpha}(0) B, \quad B A_{\alpha \ell}=A_{\alpha \ell} B \quad(\alpha \in L, 1 \leq \ell \leq g),
$$

then $B \mu_{\alpha}(z)=\mu_{\alpha}(z) B(\alpha \in L)$, because from Proposition 1

$$
\mu_{\alpha}(z)=\mu_{\alpha}(0) \exp \left\{\sum_{\ell} A_{\alpha \ell} z_{\ell}\right\} \quad(\alpha \in L) .
$$

Since $E_{\mu}$ is simple, such a matrix $B$ must be a scaler matrix. This means that $G$ is an irreducible $n \times n$-matric group. Let $V$ be the vector space of dimension non which $G$ acts. Applying Clifford theorem to the pair $\left(G, G_{0}\right)$, we get a system of inequivalent representations $\chi, \cdots, \chi_{n}$ of degree one of the abelian group $G_{0}$ such that the irreducible $G$-module $V$ decomposed into a direct sum of $G_{0}$-modules

$$
V=V_{1} \oplus \cdots \oplus V_{r}
$$

with properties that

$$
\operatorname{dim} V_{i}=\frac{n}{r}(1 \leq i \leq r)
$$




$$
\left.g_{0} v_{i}=\chi_{i}\left(g_{0}\right) v_{i} \quad g_{0} \in G_{0}, v_{i} \in V_{i}\right) .
$$

since $\chi_{1}, \cdots, \chi_{r}$ are inequivalent each other, the subspaces $V_{1}, \cdots, V_{r}$ are also $G_{1}$-modules. Moreover, since $G_{0}$ is normal in $G, G$ acts on the set $\{1, \cdots, r\}$ as follows

$$
g V_{i}=V_{i} \sigma(g) \quad(g \in G),
$$

and the kernel of $\sigma$ is $G_{1}$. Since $V$ is an irreducible $G$-module and $G / G_{1}$ is abelian, by virtue of Lemma 1 we have $\left|G / G_{1}\right|=|\sigma(G)|=r$. Next we shall show that $V_{i}(1 \leq i \leq g)$ are irreducible $G_{1}$-modules. We assume for a moment that at least one of $G_{1}$-modules of $\left\{V_{1}, \ldots, V_{r}\right\}$ is reducible, then dimension of the linear hull of $G_{1}$ over $C$ is less than

$$
r\left(\frac{n}{r}\right)^{2}=\frac{n^{2}}{r}
$$

Hence the dimension of the linear hull of $G$ is less than $r \cdot n^{2} / r=n^{2}$. This contradicts with the irreducibility of $G$-module $V$. Hence $V_{1}, \ldots, V_{r}$ are irreducible $G_{1}$-modules. From equations

$$
A_{\alpha+\beta, \ell}=u_{\alpha}(0)^{-1} A_{\beta \ell} \mu_{\alpha}(0)+A_{\alpha \ell}=\mu_{\beta}(0)^{-1} A_{\alpha \ell} \mu_{\beta}(0)+A_{\beta \ell}
$$

we observe that

$$
\mu_{\beta}(0)^{-1} A_{\alpha \ell} \mu_{\beta}(0)=A_{\alpha \ell} \quad(\alpha \in L, 1 \leq \ell \leq g),
$$

if and oly if

$$
\mu_{\alpha}(0)^{-1} A_{\beta \ell} \mu_{\alpha}(0)=A_{\beta \ell} \quad(\alpha \in L, 1 \leq \ell \leq g) .
$$

This means that $L_{1}=\left\{\beta \mid A_{\beta \ell}(1 \leq \ell \leq g)\right.$ are scaler matrices $\}$. This means that there exist a constant matrix $F$ and an irreducible representation $\rho$ of $G_{1}$ such that

$$
F^{-1} h F=\left(\begin{array}{llll}
\rho(h) & & & \\
& \rho\left(g_{1}^{-1} h g_{1}\right) & & \\
& & \ddots & \\
& & & \rho\left(g_{r}^{-1} h g_{r}\right)
\end{array}\right) \quad\left(h \in G_{1}\right),
$$

where $g_{i}=\mu_{\alpha_{i}}(0)(1 \leq i \leq r)$ and $\left\{\alpha_{1}, \cdots, \alpha_{r}\right\}$ is a system of representatives of $G / G_{1}$ in $G$ such that $1^{\sigma\left(\mu_{\left.\alpha_{i}(0)\right)}\right.}=i(1 \leq i \leq r)$. Since $\rho\left(g_{0}\right)\left(g_{0} \in G_{0}\right)$ are scaler $n / r \times n / r$-matrices, the set $\left\{\rho\left(\mu_{\beta}(0)\right) \mid \beta \in L_{1}\right\}$ is an irreducible set. In the previous note [1] we proved that a holomorphic $k \times k$-auto- 
morphic factor $\left\{\nu_{\beta}(z) \mid \beta \in N\right\}$ with respect to a lattice $N$ is written

$$
\nu_{\beta}(z)=\nu_{\beta}(0) \xi_{\beta}(z) \quad(\beta \in N)
$$

with scaler functions $\xi_{\beta}(z)$ and $\left\{\nu_{\beta}(0) \mid \beta \in N\right\}$ is an irreducible set of $k \times k$ matrices, then there exist a sublattice $N_{0}$ such that $\left[N: N_{0}\right]=k$ and $\nu_{r}(z)\left(\gamma \in N_{0}\right)$ are scaler matrices. Hence putting

$$
\begin{aligned}
& \nu_{\beta}(0)=\rho\left(\mu_{\beta}(0)\right), \quad A_{\beta \ell}=a_{\beta \ell} I_{n}, \\
& \nu_{\beta}(z)=\nu_{\beta}(0) \exp \left\{2 \pi \sqrt{-1} \sum a_{\beta \ell} z_{\ell}\right\} \quad\left(\beta \in L_{1}\right),
\end{aligned}
$$

we find a sublattice $M$ such that

$$
[L: M]=\left[L: L_{1}\right]\left[L_{1}: M\right]=r \cdot \frac{n}{r}=n
$$

and $\nu_{r}(z)(\gamma \in M)$ are scaler matrices. Since $\rho\left(\mu_{\gamma}(0)\right)(\gamma \in M)$ are scaler matrices, $\rho\left(\mu_{\alpha_{i}}(0)^{-1} \mu_{\gamma}(0) \mu_{\alpha_{i}}(0)\right)(\gamma \in M ; 1 \leq i \leq r)$ are also scaler matrices. This means that there exists holomorphic scaler automorphic factors $\left\{\eta_{r}^{(1)}(z)\right\}, \cdots,\left\{\eta_{r}^{(n)}(z)\right\}$ with respect to the lattice $M$ such that

$$
F^{-1} \mu_{r}(z) F=\left(\begin{array}{lll}
\eta_{r}^{(1)}(z) & & \\
& \ddots & \\
& \eta_{r}^{(n)}(z)
\end{array}\right) \quad(\gamma \in M)
$$

and $[L: M]=n$. Let $\mathscr{L}^{(1)}, \cdots, \mathscr{L}^{(n)}$ be line bundles over $M$ corresponding to $\left\{\eta_{r}^{(1)}(z), \cdots,\left\{\eta_{r}^{(n)}(z)\right\}\right.$, respectively. Then we have isomorphisms of vector bundles

$$
\begin{aligned}
\varphi^{*}\left(E_{\mu}\right) & \sim \mathscr{L}^{(1)} \oplus \cdots \oplus \mathscr{L}^{(n)}, \\
\varphi_{*} \varphi^{*}\left(E_{\mu}\right) & \sim \varphi_{*}\left(\mathscr{L}^{(1)}\right) \oplus \cdots \oplus \varphi_{*}\left(\mathscr{L}^{(n)}\right),
\end{aligned}
$$

where $\varphi^{*}\left(E_{\mu}\right)$ is the reciprocal image of $E_{\mu}$ and $\varphi_{*}(\mathscr{L})$ is the direct image of $\mathscr{L}$ with respect to $\varphi$. Hence, if we put

$$
\nu_{\beta}(0)=\rho\left(\mu_{\beta}(0)\right), \quad A_{\beta \ell}=a_{\beta \ell} I_{n}, \quad \nu_{\beta}(z)=\nu_{\beta}(0) \exp \left\{\sum a_{\beta \ell} z_{\ell}\right\},
$$

by virtue of the above result, we find a sublattice $M$ such that $[L: M]$ $=\left[L: L_{1}\right]\left[L_{1}: M\right]=r \cdot n / r=n$ and $\nu_{\gamma}(z)(\gamma \in M)$ are scaler $n / r \times n / r$ matrices. Since $\rho\left(\mu_{\gamma}(0)\right)(\gamma \in M)$ are scaler matrices, $\rho\left(\mu_{\alpha_{i}}(0)^{-1} \mu_{r}(0) \mu_{\alpha_{i}}(0)\right)$ $(\gamma \in M ; 1 \leq i \leq r)$ are also scaler matrices. This means that there exists holomorphic scaler automorphic factors $\left\{\eta_{r}^{(1)}(z) \mid \gamma \in M\right\}, \cdots,\left\{\eta_{r}^{(r)}(z) \mid \gamma \in M\right\}$ such that 


$$
F^{-1} \mu_{r}(z) F=\left(\begin{array}{ccc}
\eta_{r}^{(1)}(z) I_{n / r} & 0 \\
& \ddots & \\
0 & & \eta_{r}^{(r)}(z) I_{n / r}
\end{array}\right) \quad(\gamma \in M)
$$

Let $\mathscr{L}^{(1)}, \cdots, \mathscr{L}^{(r)}$ be the line bundles over $C^{g} / M$ associated to $\left\{\eta_{r}^{(1)}(z) \mid \gamma \in M\right\}$, $\cdots,\left\{\eta_{r}^{(r)}(z) \mid \gamma \in M\right\}$, respectively. Then we have an isomorphism of vector bundles

$$
\begin{aligned}
\varphi_{*} \varphi^{*}\left(E_{\mu}\right) \sim(\varphi \varphi_{*} \overbrace{\left(\mathscr{L}^{(1)}\right) \oplus \cdots \oplus \varphi \varphi_{*}}^{n / r}\left(\mathscr{L}^{(1)}\right)) \\
\oplus \cdots \oplus\left(\varphi \varphi_{*}(\overbrace{\left.\mathscr{L}^{(n)}\right) \oplus \cdots \oplus \varphi \varphi_{*}\left(\mathscr{L}^{(n)}\right)}^{n / r})\right.
\end{aligned}
$$

where $\varphi^{*}\left(E_{\mu}\right)$ is the inverse image of $E_{\mu}$ and $\varphi_{*} \varphi^{*}\left(E_{\mu}\right)$ is the direct image of $\varphi^{*}\left(E_{\mu}\right)$. Since $E_{\mu}$ is simple and $\operatorname{rank} \varphi_{*}\left(\mathscr{L}_{*}{ }^{(i)}\right)=\operatorname{rank} E_{\mu}(1 \leq i \leq n)$, $E_{\mu}$ must be isomorphic to one of $\varphi_{*}\left(\mathscr{L}^{(i)}\right)(1 \leq i \leq n)$. This completes the proof of Theorem.

THEOREM 2. Let $\left\{\mu_{\alpha}(z) \mid \alpha \in L\right\}$ be a holomorphic $n \times n$-automorphic factor with respect to a lattice $L$ in $C^{g}$, such that i) the associated vector bundle $E_{\mu}$ is simple and ii) $\mu_{\alpha}(z+\beta) \mu_{\alpha}(z)^{-1}(\alpha, \beta \in L)$ are constant matrices. Then there exist a sublattice $M$ of $L$ and a line bundle $\mathscr{L}$ on $C^{g} / M$ such that $E_{\mu}$ is isomorphic to the direct image of $\mathscr{L}$ with respect to the natural isogeny $C^{g} / M \rightarrow C^{g} / N$.

Proof. This an immediate consequence from Theorem 1 and Proposition 1.

\section{REFERENCES}

[1] H. Morikawa, A note on holomorphic vector bundles over complex tori, Nagoya Math. J. Vol. 41 (1970), 101-106.

Department of Mathematics

Nagoya University 